\title{
The potential role of FoxO transcription factor during postembryonic periods in the silkworm, Bombyx mori (Lepidoptera: Bombycidae)
}

\author{
Jin Hee KIM ${ }^{1}$, JAe-Sun CHOI ${ }^{2}$, Mi Young KIM ${ }^{1}$, Hwa Young SONG ${ }^{1}$ and Bong Hee LEE ${ }^{1,2 *}$ \\ ${ }^{1}$ School of Life Sciences and Biotechnology, Korea University, Seoul 136-701, Korea \\ ${ }^{2}$ Korean Entomological Institute, Korea University, Seoul 136-701, Korea
}

Key words. FoxO transcription factor, Bombyx mori, insulin signalling, MAPK pathways, starvation

\begin{abstract}
The Forkhead box O (FoxO) transcription factors, including FoxO1, FoxO3a, and FoxO4, have been implicated in the regulation of several biological processes, including stress resistance, metabolism, and apoptosis. In the present study, FoxO1 and FoxO3a patterns and their role in the regulation of the insulin signalling and mitogen-activated protein kinase (MAPK) pathways were analyzed after starvation in the fat body cells of the silkworm, Bombyx mori. FoxO1 and FoxO3a are localized to the nuclei. It was found that the levels of the insulin receptor and phosphoryated kinase Akt (p-Akt) increased when the animals ceased feeding. Starvation conditions caused a decrease in extracellular-signal-regulated kinase (ERK) phosphorylation, and an increase in c-Jun N-terminal kinase (JNK) and p38 (MAP kinase) phosphorylation. This implies that the FoxO transcription factors are activated by starvation and that starvation leads to changes in the insulin signalling and MAPK pathways in $B$. mori. These results strongly suggest that the FoxO transcription factor may be involved in the regulation of the insulin signalling and MAPK pathways in $B$. mori. As such, the findings provide molecular entomologists with valuable information on the molecular mechanism of the signalling pathways in postembryonic development ofthe silkworm.
\end{abstract}

\section{INTRODUCTION}

Forkhead box $\mathrm{O}$ (FoxO) transcription factors play a critical role in regulating cell growth, survival, apoptosis, differentiation and proliferation, metabolism, cell cycle arrest, and protection from oxidative stress in a variety of cell types (Accili \& Arden, 2004). The transcriptional activity of these transcription factors is regulated by multiple post-translational mechanisms. The FoxO subfamily of transcription factors consists of FoxO1, FoxO3a, and FoxO4, which are inhibited by growth factors, such as insulin and insulin-like growth factor-1 (Huang \& Tindall, 2007). In conditions involving limited supply of these growth factors, the inhibition of FoxO activity by PI3K/Akt (phosphoinositide 3-kinase/serine threonine protein kinase) is relieved, and the FoxO transcription factors are activated and accumulate in the cell nucleus (Skurk et al., 2005). Under starvation conditions, FoxO translocates into the nucleus and participates in the transcriptional activation of various genes involved in metabolism. Addition of insulin results in FoxO translocation out of the nucleus and into the cytoplasm. The insulin-signalling pathway is an important mechanism for sensing nutritional signals in organisms ranging from the nematode, Caenorhabditis elegans (Maupas) to humans (Britton et al., 2002). Insulin signalling can affect transcription patterns via regulation by FoxO transcription factors, which are inhibited by the presence of insulin (Burgering \& Medema, 2003). In Drosophila, FoxO is required for the starvation-dependent upregulation of the insulin receptor (Puig \& Tjian, 2005). Akt (also known as protein kinase $\mathrm{B}[\mathrm{PKB}]$ ) plays a critical role in mediating various effects of insulin and related growth factors downstream of PI3K. Akt is an important effector molecule in the insulin-signalling pathway because it phosphorylates glucose transporters and glycogen synthase kinase to promote glucose absorption and glycogen synthesis (Taniguchi et al., 2006). This also phosphorlyates and inhibits the activity of FoxO transcription factors (Barthel et al., 2005).

Three major groups of mitogen-activated protein kinases (MAPK) are found in mammalian cells: extracellular signal-regulated protein kinase (ERK), p38 MAPK, and c-Jun N-terminal kinase (JNK). MAP kinases regulate many cellular activities, ranging from gene expression to mitosis, movement, metabolism, and apoptosis (Davis, 2000). These MAP kinases are activated by dual phosphorylation of neighbouring threonine and tyrosine residues in response to various extracellular stimuli (Kyriakis \& Avruch, 1996). In particular, p38 and JNK are implicated in stress-responsive signalling leading to the initiation of adaptive events such as gene differentiation and apoptosis (Ono \& Han, 2000). ERKs are activated by growth signals such as epidermal growth factor (Lubinus et al., 1994).

FoxO transcription factors also play a crucial role in the regulation of tissue homeostasis in organs and in complex diseases. Despite extensive studies on the functional, cellular, and molecular aspects of FoxO transcription factors, the cellular production, molecular characteristics, and functional aspects of these factors in the silkworm, Bombyx mori, remained poorly understood until recently.

\footnotetext{
* Corresponding author; e-mail: bhlee@korea.ac.kr
} 
In the present study, and in light of the aforementioned previous findings FoxO1 and FoxO3a, and their regulation of the insulin signalling and MAPK pathways were investigated in the $B$. mori fat body following starvation of the larval stages of the insect.

\section{MATERIAL AND METHODS}

\section{Animals}

Cold-treated eggs of the silkworm, Bombyx mori L. (Lepidoptera: Bombycidae), were obtained from the National Institute of Agricultural Science and Technology, Suweon, South Korea. The eggs were incubated in plastic containers for about ten days at $26.5^{\circ} \mathrm{C}$ following their hatching; the time and date of hatching were recorded. The larvae were reared on the Silkmate ${ }^{\mathrm{TM}}$ artificial diet (Nihon Nosan Kogyo, Yokohama, Japan) and incubated at $25^{\circ} \mathrm{C}$ with alternating $12 \mathrm{~h}$ periods of light and dark, and $70 \%$ humidity in growth chamber (Doori, Seoul, Korea). Fifth instar larvae were used during the starvation experiment. The starved larvae were reared under the same conditions without food and placed in Tupperware plastic containers containing a moist paper towel.

\section{Immunohistochemistry and microscopy analysis}

Tissue preparation and whole mount immunohistochemistry were performed as described by $\mathrm{Na}$ et al. (2004). After anesthetic treatment of stage- 5 pupae at $4^{\circ} \mathrm{C}$ for $1 \mathrm{~h}$, the fat bodies were isolated in $0.1 \mathrm{M}$ sodium phosphate buffer (PB), $\mathrm{pH} 7.4$, and fixed in $4 \%$ paraformaldehyde in $0.1 \mathrm{M} \mathrm{PB}$ for $6 \mathrm{~h}$ at $4{ }^{\circ} \mathrm{C}$. The fixed tissues were immersed in $0.1 \mathrm{M}$ phosphate-buffered saline with $1 \%$ Triton X-100 (PBST) at $4{ }^{\circ} \mathrm{C}$ overnight. Peroxidase activity was blocked by dipping the tissues in a $3 \% \mathrm{H}_{2} \mathrm{O}_{2}$ solution with $10 \%$ methanol for $25 \mathrm{~min}$. This was followed by washing of the tissues in $0.1 \mathrm{M}$ Tris- $\mathrm{HCl}$ buffer ( $\mathrm{pH} 7.6-8.6$ ) containing $1 \%$ Triton $\mathrm{X}-100$ and $4 \% \mathrm{NaCl}$. The tissue samples were shaken gently and incubated with either anti-rabbitpolyclonal phospho-FoxO1A (Abcam, Cambridge, MA, USA; product No. 38501) or anti-rabbit-polyclonal phospho-FoxO3A (Abcam; product No. 47285), both of which were diluted to $1: 200$ with a dilution buffer ( $0.1 \mathrm{M}$ PBST and $10 \%$ normal goat serum), for 1 day. After washing in $0.1 \mathrm{M}$ PBST, tissues were incubated in a 1:500 dilution of goat anti-rabbit Cy3-conjugated antibody for 1 day at $4^{\circ} \mathrm{C}$. The tissues were then rinsed in 0.005 $\mathrm{M}$ Tris- $\mathrm{HCl}$ buffer, mounted in glycerin, examined, and photographed using a model LSM 310 microscope (Carl Zeiss, Jena, Germany).

\section{Western blotting}

Larvae were dissected under a stereoscope, and fat bodies isolated in $0.1 \mathrm{M} \mathrm{PB}(\mathrm{pH} 7.4)$. Isolated tissues were washed in fresh PB buffer, homogenized in PB buffer, and then centrifuged at $500 \times \mathrm{g}$ for $3 \mathrm{~min}$ and then supernatant was removed. For nuclear extracts, the cells were lysed using NE-PER extraction reagent (Pierce, Rockland, IL, USA) according to the manufacturer's protocol.

The pellets were layered over $200 \mu 1$ of Cytoplasmic Extract Reagent I (CER I) and incubated on ice for ten min. The pellets were layered over $11 \mu \mathrm{l}$ of Cytoplasmic Extract Reagent II (CER II) and incubated on ice for $1 \mathrm{~min}$. The supernatant solutions were then combined and centrifuged at $16,000 \times \mathrm{g}$ for 5 min. The resulting supernatant fraction contained cytoplasmic extract. The insoluble fractions were added to $100 \mu 1$ of Nuclear Extraction Reagent (NER) and incubated on ice for $40 \mathrm{~min}$. The solutions were then combined and centrifuged at $16,000 \times \mathrm{g}$ for $10 \mathrm{~min}$. The resulting supernatant fraction contained nuclear extract.
Protein concentration was determined by Bradford assay. The purified proteins were separated by polyacrylamide gel electrophoresis (SDS-PAGE) and the resolved proteins transferred to a nitrocellulose membrane. Each membrane was incubated overnight with the primary antibody at $4{ }^{\circ} \mathrm{C}$. The primary antibodies included anti-rabbit-polyclonal phospho-FoxO1 (Abcam; product No. 38501), anti-rabbit-polyclonal phospho-FoxO3A (Abcam; product No. 47285), anti-rabbit-polyclonal phospho-Akt (Cell Signaling Technology, Danvers, MA, USA; product No. 9271), anti-rabbit-polyclonal Akt (Cell Signaling Technology; product No. 9272), anti-rabbit-polyclonal insulin receptor (Abcam; product No. 5500), anti-rabbit-polyclonal phospho-ERK (Cell Signaling Technology; product No. 9101), antirabbit-polyclonal ERK (Cell Signaling Technology; product No. 9102), anti-rabbit-polyclonal phospho-p38 (Cell Signaling Technology; product No. 9211), anti-rabbit-polyclonal p38 (Cell Signaling Technology; product No. 9212), anti-mousemonoclonal Actin (Abcam; product No. 3280) and anti-mousemonoclonal TATA-binding protein TBP (Abcam; product No. 818 ) at dilutions of 1:1000 and 1:500. The membranes were treated with a 1:1000 dilution of peroxidase-conjugated secondary anti-rabbit or anti-mouse antibodies for $2 \mathrm{~h}$. The proteins were detected using the enhanced chemiluminescence (ECL) western blotting method (Amersham Biosciences, Piscataway, NJ, USA). Densitometric quantification of the bands was performed using ImageJ software (version 1.29x: NIH, Bethesda, MD, USA) (Abramoff et al., 2004).
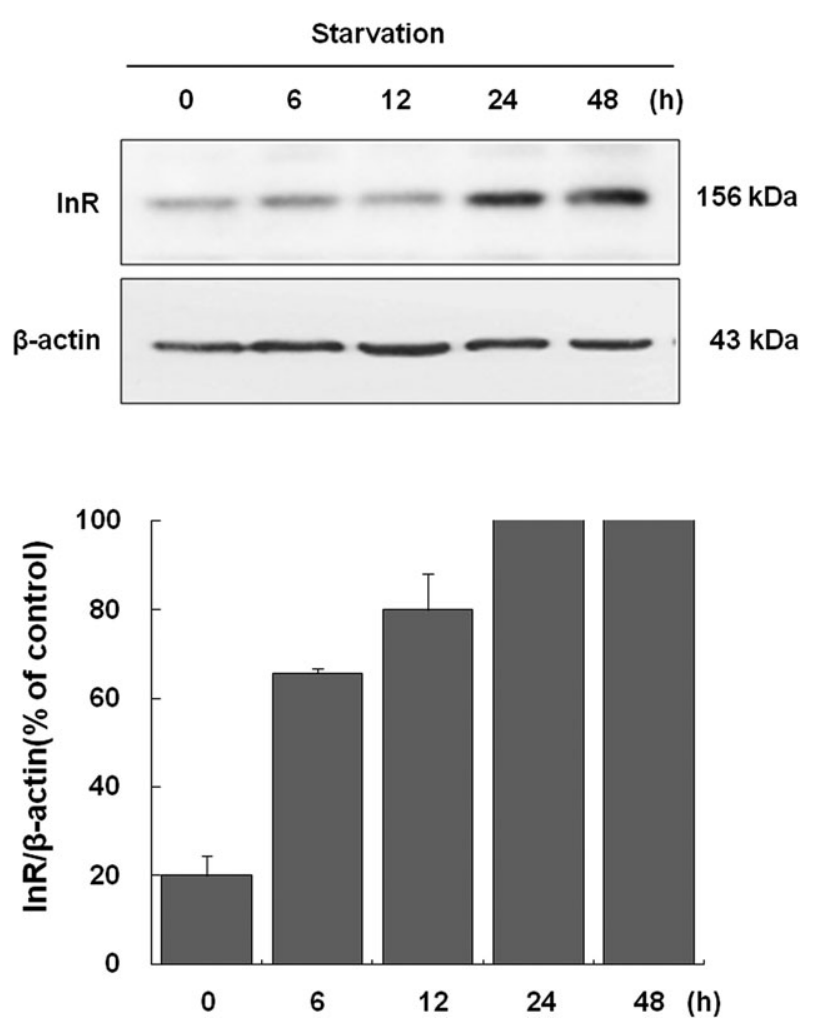

Fig. 1. Western blots of fat body from Bombyx mori 5th instar larvae showing existence of anti-rabbit-polyclonal InR. The level of InR gradually increased after starvation. $\beta$-actin was served as a control. Densitometric quantification of the bands was performed using ImageJ software (version 1.29x: NIH, Bethesda, MD). Error bars represent the mean $( \pm \mathrm{SD})$ of three replicates. 

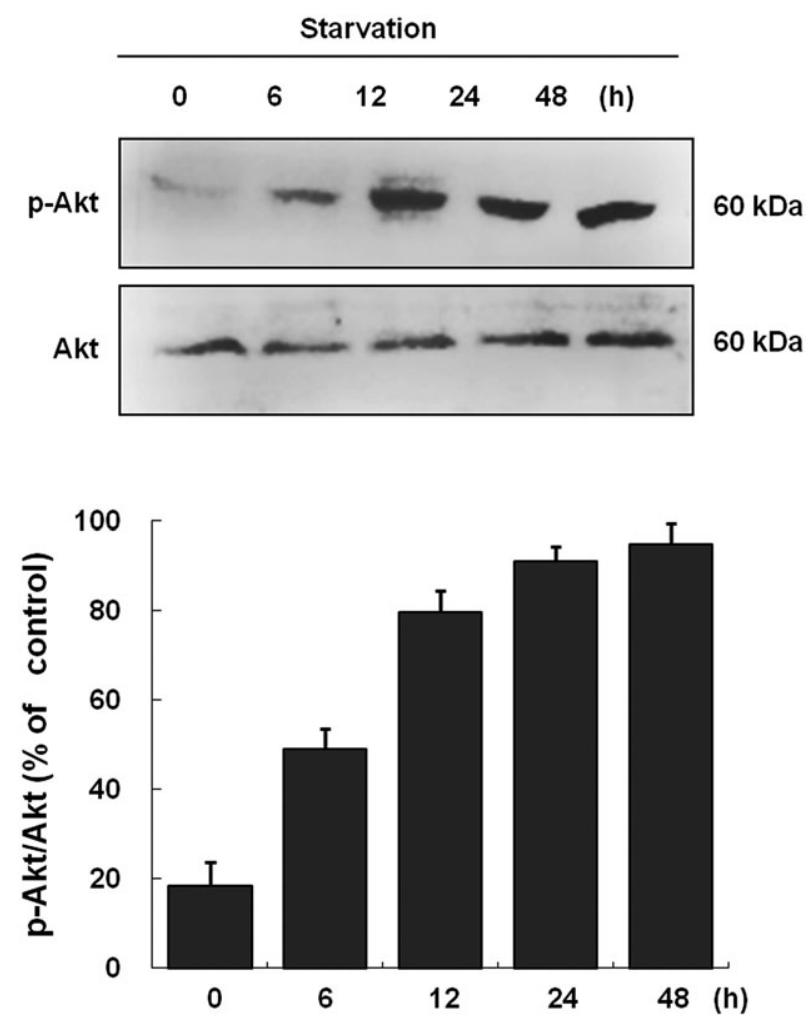

Fig. 2. Western blots of fat body from Bombyx mori 5 th instar larvae showing existence of $\mathrm{p}$-Akt and Akt. The level of p-Akt gradually increased after starvation. Densitometric quantification of the bands was performed using ImageJ software (version 1.29x: NIH, Bethesda,MD). Error bars represent the mean $( \pm \mathrm{SD})$ of three replicates.

\section{Statistical analyses}

Results are expressed as mean $\pm \mathrm{SD}$. Comparisons between groups were made by one-way analysis of variance (ANOVA) followed by Tukey's multiple comparison test, with $P$ values of $<0.05$ considered statistically significant.

\section{RESULTS}

To determine the change of $B$. mori insulin signalling, protein in the fat body from day 2 of 5 th instar larvae were analyzed $6,12,24$, and $48 \mathrm{~h}$ after starvation. The effect of starvation on activation of the insulin receptor and p-Akt level was measured. Results from Western blot showed that insulin receptor and p-Akt levels markedly increased $12 \mathrm{~h}$ after starvation (Figs 1,2 ).

To assess the roles of the phosphorylation status of FoxO1 and FoxO3a, changes in phosphorylated FoxO protein levels were measured after starvation. Changes in nuclear localization were investigated by Western blot analysis. Because FoxO proteins were partially regulated by control of nuclear localization, the nuclear and cytoplasmic distributions of FoxO1 and FoxO3a were measured after starvation. From day 2, protein levels in the fat body of the 5 th instar larvae were analyzed at $6,12,24$, and $48 \mathrm{~h}$ after starvation and compared with the corresponding levels in normally fed insects. Increase in the starvation period from 0 to $6 \mathrm{~h}$ had no significant effects on FoxO1 protein levels, which increased in the nucleus only after $12 \mathrm{~h}$ of starvation. However, cytoplasmic levels of FoxO1 decreased after $24 \mathrm{~h}$ of starvation. FoxO3a protein levels in the nucleus increased $12 \mathrm{~h}$ after starvation, whereas its cytoplasmic levels decreased after $24 \mathrm{~h}$ of
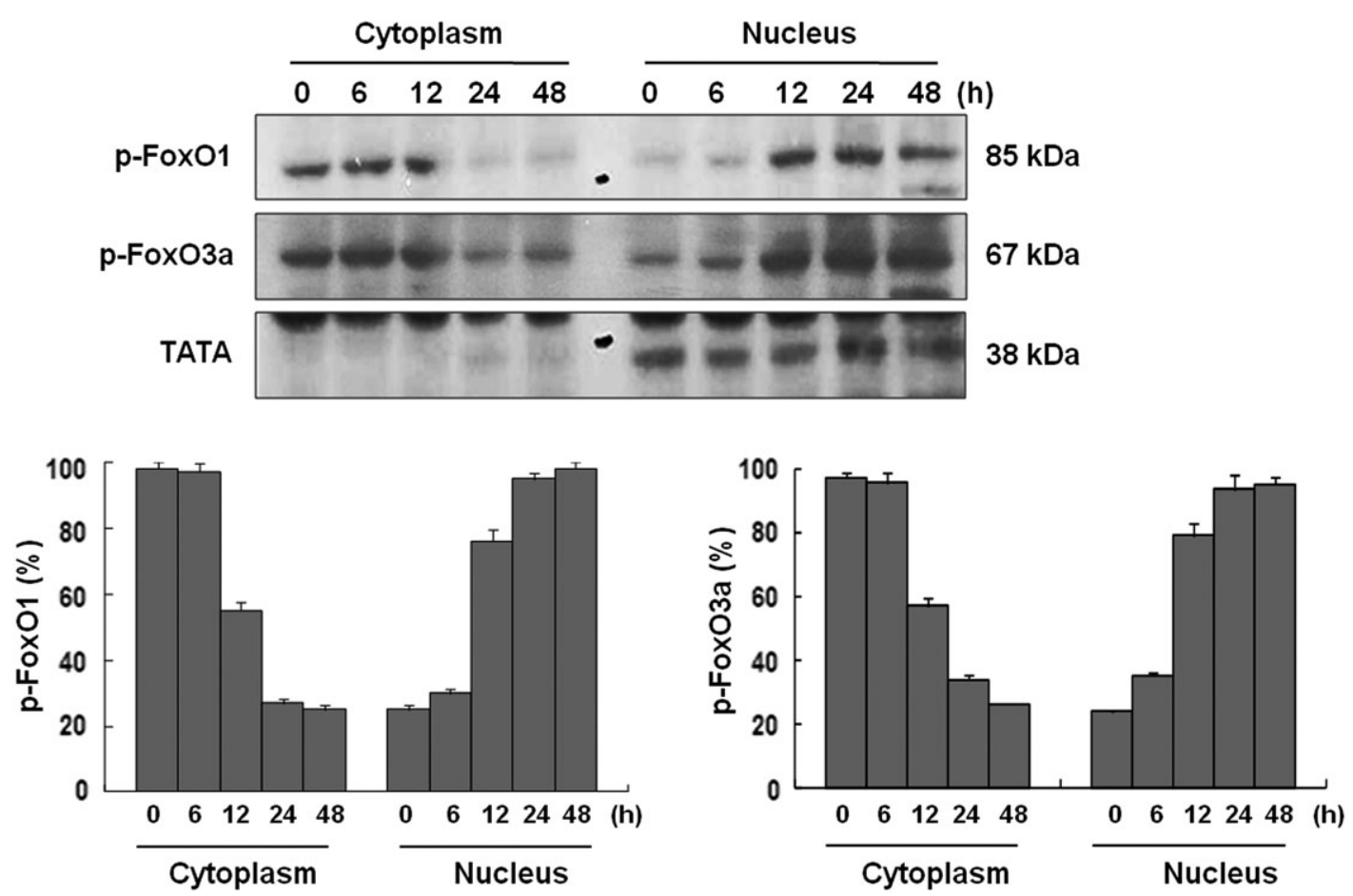

Fig. 3. Western blots of fat body from Bombyx mori 5th instar larvae showing localization of p-FoxO1 and p-FoxO3a. Cytoplasm and nucleus extracts were prepared from fat body under starvation conditions. Levels of p-FoxO1 and p-FoxO3a gradually decreased in the cytoplasm after starvation, but levels of p-FoxO1 and p-FoxO3a increased in the nucleus after starvation. TATA-binding protein was used as a nucleus marker. Densitometric quantification of the bands was performed using ImageJ software (version 1.29x: $\mathrm{NIH}$, Bethesda, MD). Error bars represent the mean $( \pm \mathrm{SD})$ of three replicates. 

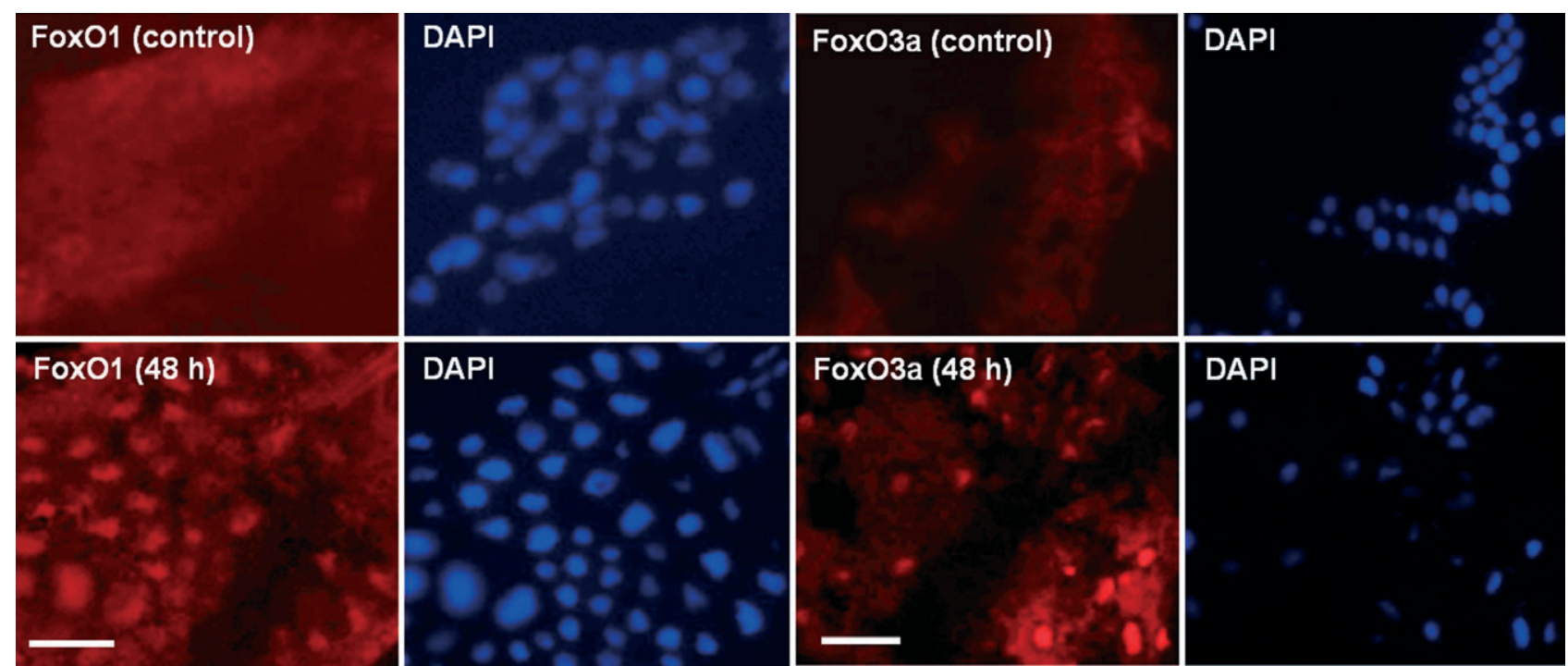

Fig. 4. Immunohistochemistry of fat body cells showing localization of p-FoxO1 and p-FoxO3a. The fat body isolated from Bombyx mori 5th instar larvae under starvation conditions were analyzed using p-FoxO1 and p-FoxO3a antibodies (red) and DAPI (blue), showing the location of the nuclei $(48 \mathrm{~h})$, respectively. Fat droplets in these cells generated a mesh-like appearance in the cytoplasm (control). Scale bar indicates $50 \mu \mathrm{m}$.
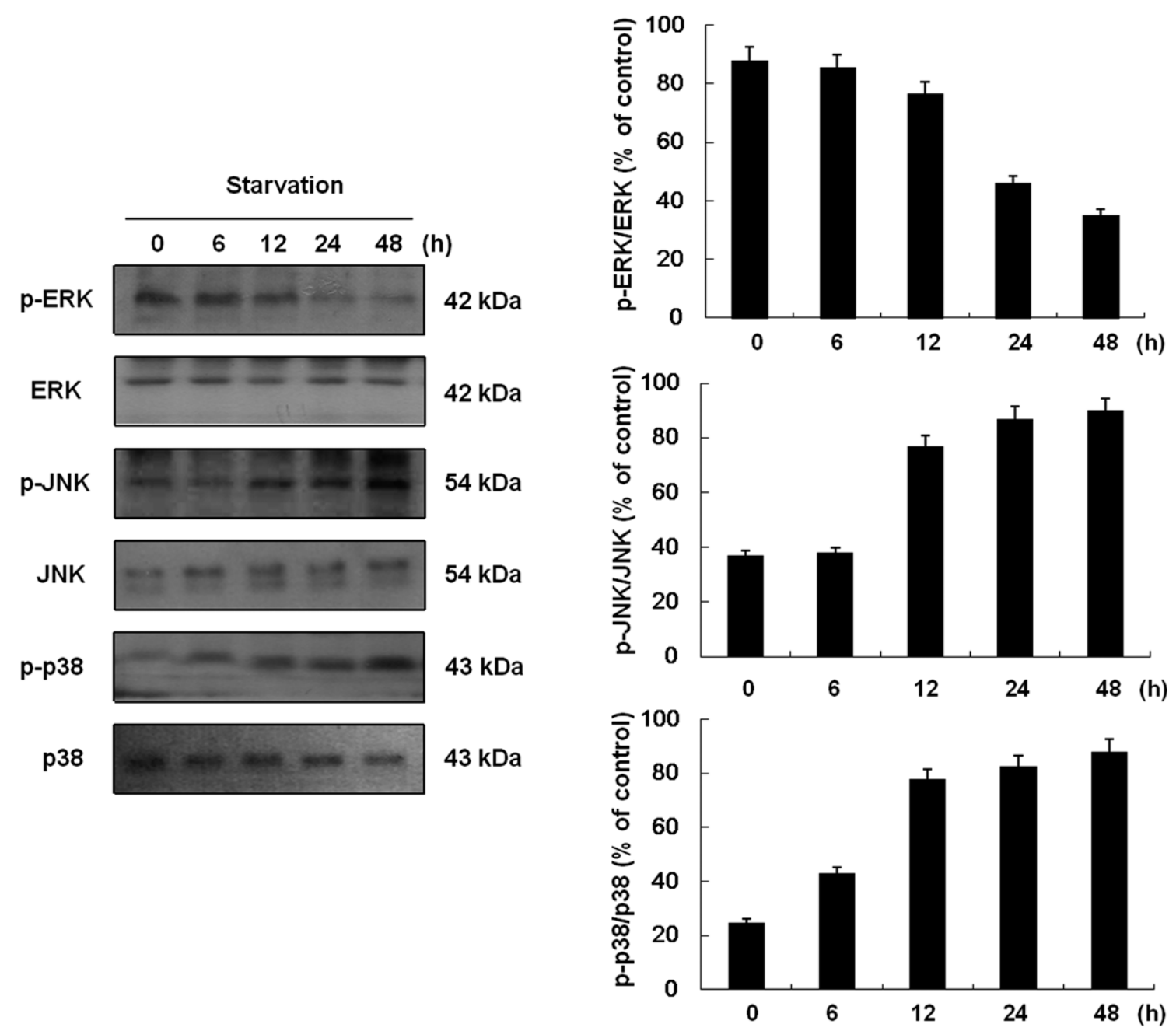

Fig. 5. Western blots of fat body showing existence of p-ERK, ERK, p-JNK, JNK, p-p38 and p38. The level of p-ERK decreased after starvation, but the level of p-JNK and p-p38 increased after starvation. Densitometric quantification of the bands was performed using ImageJ software (version 1.29x: NIH, Bethesda, MD). Error bars represent the mean $( \pm \mathrm{SD})$ of three replicates. 
starvation (Fig. 3). The changes in the FoxOl and FoxO3a after nuclear localization were investigated by immunohistochemistry and also studied during nuclear localization of these factors after starvation. Compared to the fat body samples obtained from normally fed larvae, the samples obtained from starved 5th instar larvae (starved for $48 \mathrm{~h}$ ) showed increased nuclear translocation of FoxO1 and FoxO3a (Fig. 4).

MAPK pathways regulate many cellular activities ranging from gene expression to metabolism (Farooq \& Zhou, 2004). The change of ERK, JNK and p38 MAPKs was examined during starvation (Fig. 5). The starvation condition caused a decrease in ERK phosphorylation and an increase in JNK phosphoryaltion and p38 phosphorylation. These data suggest that starvation might inhibit growth and induce apoptosis through regulation of MAPK pathways in $B$. mori.

\section{DISCUSSION}

The insulin-signalling pathway in insects is of major interest as it is conserved in animals, both in terms of structure and function. Bombyxin is an insect neurohormone with an insulin-like peptide structure. The first Bombyxin was identified from B. mori about 30 years ago (Nagasawa et al., 1984). Similar to mammal insulin, Bombyxin regulates sugar and lipid metabolism in the insect (Masumura et al., 1999; Satake et al., 1999). Remarkably, Bombyxin stimulates the phosphorlyation of insulin receptor and Akt, in a manner apparently identical to mammalian insulin (Nagata et al., 2008). In Drosophila, there are seven insulin-like peptides that are expressed in several tissues. A single insulin receptor of the receptor tyrosine kinase family mediates the action of the seven insulin-like peptides. Once bound by the insulin-like peptides, the insulin receptor is selfphosphorylated and auto-activated (Brogiolo et al., 2001). Therefore, an increase in the number of insulin receptors in response to starvation would increase receptor sensitivity when the nutrient availability is restored.

The fat body is the central organ that integrates and coordinates different hormonal and nutritional signals to regulate growth and final body size in Drosophila melanogaster (Meigen) (Colombani et al., 2005). Inhibition of the Drosophila insulin-signalling pathway in the fat body induces the cellular and organismal effects of starvation, whereas its activation in a small portion of fat body cells with an Flp-out mosaic method bypasses the nutrient requirement for cell growth. Activity of the insulinsignalling pathway in the fat body is regulated by the availability of dietary protein in vivo (Britton et al., 2002).

The FoxO transcription factor can be phosphorylated by Akt, inhibited by the insulin-signalling pathway, and activated by starvation. Overexpression of FoxO reduces the body size and induces starvation in Drosophila (Kramer et al., 2003). Under the conditions of starvation, FoxO transcription factor activates the insulin receptor mRNA expression (Puig et al., 2003) by directly binding to the FoxO response element in the insulin receptor promoter region (Puig \& Tjian, 2005).

The FoxO transcription factor is an important target of insulin and its growth factor activity. FoxO transcription factors show both nuclear localization and nuclear export signals, and are shuttled between the cytoplasm and nucleus. Typically, FoxO transcription factors are localized in the cytoplasm, which restricts their transcriptional activation activity. On activation, the FoxO transcription factors are re-located to the nucleus. However, the mechanisms regulating nuclear/cytoplasmic shuttling of FoxO transcription factors remained poorly understood until recently.

It has previously been reported that the concentration of amino acid is altered by changes in the nutritional status (Fafournoux et al., 1990). The present study demonstrated a signal transduction pathway in $B$. mori that responds to variations in the starved state. This study also showed that starvation possibly causes the activation of JNK/p38 and the inhibition of ERK in B. mori. Therefore, ERK activation appears to be protected after stress and is dependent upon both JNK and p38.

The FoxO transcription factors changes its intracellular localization from the cytoplasm to nucleus under stress conditions (Kaneto et al., 2004). The overexpression of JNK induced the nuclear localization of FoxO transcription factors whilst the suppression of JNK reduced the nuclear localization of FoxO transcription factors, suggesting involvement of the JNK pathway in FoxO translocation. Activation of ERK has been shown to phosphorylate FoxO transcription factors, resulting in nuclear exclusion and transcriptional repression. In addition to ERK, phosphorylation of FoxO transcription factors by Akt results in cytoplasmic retention, inhibiting the expression of FoxO transcription factors, which control the cell death and stress (Stahl et al., 2002).

In conclusion, FoxO transcription factors are activated by starvation and the starved state leads to changes in the insulin signalling and MAPK pathways in B. mori. Our data strongly suggest that the FoxO transcriptional factor is involved in the regulation of the insulin signalling and MAPK pathways in this insect. The molecular mechanisms of the signalling pathways remain to be further elucidated.

ACKNOWLEDGEMENTS. This work was supported in part by a grant to B.H. Lee in 2011 from the Korea University.

\section{REFERENCES}

Abramoff M.D., Maelhaesm P.J. \& Ram S.J. 2004: Image processing with Image. J. Biophotonics Int. 11: 36-42.

Accili A.D. \& ARden K.C. 2004: FoxOs at the crossroads of cellular metabolism, differentiation, and transformation. Cell 117: 421-426.

Barthel A., Schmoll D. \& Unterman T.G. 2005: FoxO proteins in insulin action and metabolism. Trends Endocrin. Metab. 16: 183-189.

Britton J.S., Lockwood W.K., Li L., Cohen S.M. \& Edgar B.A. 2002: Drosophila's insulin/PI3-kinase pathway coordinates cellular metabolism with nutritional conditions. Dev. Cell 2: 239-249. 
Brogiolo W., Stocker H., Ikeya T., Rintelen F., Fernandez R. \& HAFEN E. 2001: An evolutionarily conserved function of the Drosophila insulin receptor and insulin-like peptides in growth control. Curr. Biol. 11: 213-221.

Burgering B.M. \& Medema R.H. 2003: Decisions on life and death: FoxO Forkhead transcription factors are in command when PKB/Akt is off duty. J. Leukoc. Biol. 73: 689-701.

Colombani J., Bianchini L., Layalle S., Pondevill E., DauphinVillemant C., Antoniewsik C., Carrec C., Noselli S. \& LeoPOLD P. 2005: Antagonistic actions of ecdysone and insulins determine final size in Drosophila. Science 310: 667-670.

DAVIS R.J. 2000: Signal transduction by the JNK group of MAP kinases. Cell 103: 239-252.

Fafaournoux P., Remesy C. \& Dmigne C. 1990: Fluxes and membrane transport of amino acids in rat liver under different protein diets. Am. J. Physiol. (E) 259: 614-625.

FAROOQ A. \& ZHOU M.M. 2004: Structure and regulation of MAPK phosphatases. Cell. Signal. 16: 769-779.

Huang H. \& Tindall D.J. 2007: Dynamic FoxO transcription factors. J. Cell. Sci. 120: 2479-2487.

Kaneto H., Kawamori D., Nakatani Y., Gorogawa S. \& MatSUOKA T.A. 2004: Oxidative stress and the JNK pathway as a potential therapeutic target for diabetes. Drug News Perspect. 17: 447-453.

Kramer J.M., Davidge J.T., Lockyer J.M. \& Staveley B.E. 2003: Expression of Drosophila FOXO regulates growth and can phenocopy starvation. BMC Dev. Biol. 5: 3-5.

KyriaKIS J.M. \& AvRUCH J. 1996: Sounding the alarm: protein kinase cascades activated by stress and inflammation. J. Biol. Chem. 271: 24313-24316.

Lubinus M., Meier K.E., Smith E.A., Gause K.C., Leroy E.C. \& TrojanowsKA M. 1994: Independent effects of plateletderived growth factor isoforms on mitogen-activated protein kinase activation and mitogenesis in human dermal fibroblasts. J. Biol. Chem. 269: 9822-9825.

Masumura M., Satake S.I., Saegusa H. \& Mizoguchi A. 1999: Glucose stimulates the release of Bombyxin, an insulinrelated peptide of the silkworm Bombyx mori. Gen. Com. Endocr. 118: 393-399.

Na S.Y., Sung D.K., Kim K.K., KIm K.M., Kim J.H., PARK H.H., Lee S.M., Seong S.I., Chang J.S., Hwang J.S., Kang S.W.,
KIM H.R. \& LeE B.H. 2004: FMRF-expressing efferent neurons in eighth abdominal ganglion innervate hindgut in the silkworm, Bombyx mori. Zool. Sci. 21: 805-811.

Nagasawa H., Kataoka H., Hori Y., Isogai A., Tamura S., Suzuki A., Guo F., Zhong X.C., Mizoguchi A. \& Fujishita M. 1984: Isolation and some characterization of the prothoracicotropic hormone from Bombyx mori. Gen. Comp. Endocr. 53: 143-152.

Nagata S., Hakuno F., Takahashi S. \& Nagasawa H. 2008: Identification of Bombyx mori Akt and its phosphorylation by bombyxin stimulation. Comp. Biochem. Physiol. (A) 151: 355-360.

Ono K. \& Han J. 2000: The p38 signal transduction pathway: activation and function. Cell. Signal. 12: 1-13.

PUIG O. \& TJIAN R. 2005: Transcriptional feedback control of insulin receptor by dFOXO/FOXO1. Genes Dev. 19: 2435-2446.

Puig O., Marr M.T., Ruhf M.L. \& TJian R. 2003: Control of cell number by Drosophila FOXO: downstream and feedback regulation of the insulin receptor pathway. Genes Dev. 17: 2006-2020.

Satake S., Nagata K., Kataoka H. \& Mizoguchi A. 1999: Bombyxin secretion in the adult silkmoth Bombyx mori: sexspecificity and its correlation with metabolism. J. Insect Physiol. 45: 939-945.

Skurk C., Izumiya Y., Maatz H., Razeghi P., Shiojima I., Sandri M., Sato K., Zeng L., Schiekofer S., Pimentel D., Lecker S., Taegtmeyer H., Goldberg A.L. \& Walsh K. 2005: The FOXO3a transcription factor regulates cardiac myocyte size downstream of AKT signalling. J. Biol. Chem. 280: 20814-20823.

Stahl M., Dijkers P.F., Kops G.J., Lens S.M., Coffer P.J., BurGERING B.M. \& MEDEMA R.H. 2002: The forkhead transcription factor FoxO regulates transcription of $\mathrm{p} 27 \mathrm{Kip} 1$ and Bim in response to IL-2. J. Immunol. 168: 5024-5031.

Taniguchi C.M., Kondo T., Sajan M., Luo J., Bronson R., asano T., Farese R., Cantley L.C. \& Kahn C.R. 2006: Divergent regulation of hepatic glucose and lipid metabolism by phosphoinositide 3-kinase via AKT and PKC lambda/zeta. Cell Metb. 3: 343-353.

Received December 21, 2011; revised and accepted April 4, 2012 